\title{
A small molecule interacts with pMAC-derived hydroperoxide reductase and enhances the activity of aminoglycosides
}

\author{
Zhen Hui ${ }^{1}$ Shiyi Liu ${ }^{1}$ Ruiqin Cui ${ }^{1}$ Biao Zhou ${ }^{1}$ Chunxia Hu${ }^{1} \cdot$ Min Zhang ${ }^{1}$ - Qiuyang Deng ${ }^{1}$ Shumin Cheng ${ }^{1}$.

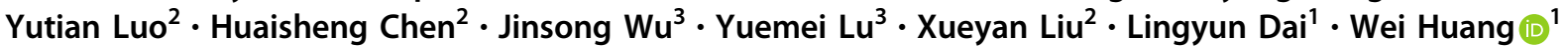

Received: 24 November 2020 / Revised: 5 December 2020 / Accepted: 12 December 2020 / Published online: 18 January 2021

(c) The Author(s), under exclusive licence to the Japan Antibiotics Research Association 2021

\begin{abstract}
The threat of antimicrobial resistance calls for more efforts in basic science, drug discovery, and clinical development, particularly gram-negative carbapenem-resistant pathogens. We sought to identify novel antibacterial agents against Acinetobacter baumannii ATCC19606 using whole cell-based screening. A small molecule named 6D1 with the chemical structure of 6-fluorobenzo[d]isothiazol-3(2H)-one was identified and exhibited activity against $A$. baumannii ATCC19606 strain (minimal inhibitory concentration, $\mathrm{MIC}=1 \mathrm{mg} \mathrm{l}^{-1}$ ). The mutation in the plasmid-derived $o h r B$ gene that encodes a peroxidase was identified in spontaneously resistant mutants. Treatment of the bacteria with $6 \mathrm{D} 1$ resulted in increased sensitivity to peroxide, such as tert-butyl hydroperoxide. The binding of 6D1 and OhrB was confirmed by surface plasmon resonance. Interestingly, the MIC of kanamycin and gentamicin against spontaneously resistant mutants decreased. Finally, we identified the effect of 6D1 on enhancing the antibacterial activity of kanamycin and gentamicin, including against New Delhi metallo$\beta$-lactamase (NDM-1)-producing carbapenem-resistant Klebsiella pneumoniae, but not in strains carrying aminoglycosides resistance genes. In this study, we identified a small molecule that suppresses the growth of A. baumannii, interacts with hydroperoxide reductase from A. baumannii ATCC19606 plasmid pMAC, and enhances the antibacterial activity of kanamycin and gentamicin. We propose that peroxidase may be potentially used as a target for aminoglycosides adjuvant development.
\end{abstract}

These authors contributed equally: Zhen Hui, Shiyi Liu

Supplementary information The online version of this article (https:// doi.org/10.1038/s41429-020-00401-2) contains supplementary material, which is available to authorized users.

\section{Wei Huang}

whuang_sz@163.com

1 Bacteriology \& Antibacterial Resistance Surveillance Laboratory, Shenzhen Institute of Respiratory Diseases, Shenzhen People's Hospital (The Second Clinical Medical College, Jinan University; The First Affiliated Hospital, Southern University of Science and Technology), Shenzhen 518020 Guangdong, China

2 Intensive Care Unit, Shenzhen People's Hospital(The Second Clinical Medical College, Jinan University; The First Affiliated Hospital, Southern University of Science and Technology), Shenzhen 518020 Guangdong, China

3 Department of Clinical Laboratory, Shenzhen People's Hospital (The Second Clinical Medical College, Jinan University; The First Affiliated Hospital, Southern University of Science and Technology), Shenzhen 518020 Guangdong, China

\section{Introduction}

Gram-negative pathogens, such as Acinetobacter baumannii, K. pneumoniae, and Pseudomonas aeruginosa have become resistant to almost all commonly used antimicrobial agents, including aminoglycosides, quinolones, and broad-spectrum $\beta$-lactams. Overall, for instance, $\sim 45 \%$ of all global $A$. baumannii isolates are considered multidrug-resistant (bacteria resistant to more than three antibiotic classes) $[1,2]$.

With the emergence of carbapenem-resistant gram-negative pathogens, such as A. baumannii (CRAB), tigecycline and polymyxin-class antibiotics are the only currently available treatment options [3]. However, treatment outcomes of tigecycline have been hampered by the low serum concentrations of the drug in the approved dosing regimen and the low penetration in the epithelial lining fluid of mechanically ventilated patients [4]. The resistance of polymyxin-class antibiotics as well as nephrotoxicity and neurotoxicity are the major factors that limit the usage of polymyxin. Previous clinical observations showed that the rates of nephrotoxicity occurred in $\sim 60 \%$ of patients who received colistin or polymyxin B therapy [5-7]. Eravacycline, cefiderocol, and 
plazomicin seem to be promising new agents against $A$. baumannii. However, evaluation of their position in clinical practice and particularly in ventilator-associated pneumonia has not been performed to date $[8,9]$.

The present clinical pipeline does not meet current needs, and thus more investment is required in basic science, drug discovery, and clinical development, particularly gramnegative carbapenem-resistant pathogens, including CRAB [10]. Therefore, we launched a whole cell-based screening program for A. baumannii. Here, we report the discovery of compound 6D1 that exhibits anti-A. baumannii activity. In addition, we show the enhancement effect of 6D1 on the antibacterial efficacy of aminoglycosides through the inhibition of plasmid-derived OhrB.

\section{Materials and methods}

\section{Bacterial strains, growth conditions, reagents, and screening strategy}

A. baumannii, K. pneumoniae, and $P$. aeruginosa were grown in liquid broth (LB) medium or LB agar. Antibiotic (purchased from Sigma-Aldrich, USA) solutions were prepared at a concentration of $1 \mathrm{mg} \mathrm{ml}^{-1}$ in distilled water or $100 \%$ dimethylsulfoxide (DMSO), filter-sterilized, and frozen at $-20^{\circ} \mathrm{C}$ until use. The 100,000 compounds from Topscience Co. to be screened were dissolved in $100 \%$ DMSO and stored as frozen stocks at a concentration of $1 \mathrm{mg} \mathrm{ml}^{-1}$.

We sought anti-A. baumannii compounds by testing compounds for inhibition of A. baumannii ATCC19606. A wholecell assay was used because of its ability to concurrently assess multiple targets. Compounds were prepared in 96-well plates at a concentration of $10 \mathrm{mg} \mathrm{l}^{-1}$ in $50 \mu \mathrm{LB}$ broth. A $50-\mu \mathrm{l}$ aliquot of each bacteria culture was then added to each well of the 96-well plate at an $\mathrm{OD}_{600}=0.006$. The plates were incubated overnight at $37^{\circ} \mathrm{C}$, and the primary active hits were filtered by achieving at least $90 \%$ of bacterial growth inhibition using Cell Counting Kit-8 (MCE, USA). Subsequently, twofold serial dilutions of primary hits were prepared for the determination of minimal inhibitory concentration (MIC), defined as the lowest concentration of compound that inhibited $90 \%$ of bacterial growth. Compounds with an MIC $\leq 1 \mathrm{mg} \mathrm{l}^{-1}$ were selected for further investigation.

\section{Spontaneously resistant mutant selection}

Spontaneously resistant mutants were selected via stepwise exposure to increasing concentrations of the compounds. An aliquot of mid-log phase $\left(\mathrm{OD}_{600}=0.6\right)$ bacterial culture $(1 \mathrm{ml})$ was added to $2 \mathrm{ml}$ of medium containing serial increasing concentrations of 6D1 until no growth was observed. The bacteria that survived in culture were spread onto agar plates containing the corresponding concentrations of the 6D1 compound. All colonies that originated from different plates and represent independent biological events were subjected to whole-genome sequencing (WGS). The resistance phenotype to the compound was confirmed by testing for a shift in MIC values.

\section{WGS}

Genomic DNA was extracted from each isolate using a gram-negative bacterial genome extraction kit (Tiangen, China). Whole-genome fragment libraries were prepared using a paired-end sample preparation kit (Illumina, USA). The genomes were sequenced using Illumina HiSeq 2500 platform (Illumina, USA) and assembled with de novo SPAdes Genome Assembler (version 3.12.0) [11]. The resulting reads were mapped to the A. baumannii ATCC19606 reference genome, and mutations were identified using Snippy (https://github.com/tseemann/snippy).

The Contig files supporting the findings of the present study have been deposited in the National Center for Biotechnology Information's Sequence Read Archive with accession number PRJNA649401.

\section{Effect of compounds on the tolerance of bacteria to peroxides}

The effects on the tolerance of bacteria to peroxides were determined by testing for shifts in MIC of tert-butyl hydroperoxide ( $t$-BHP), cumene hydroperoxide (CHP), and hydrogen peroxide $\left(\mathrm{H}_{2} \mathrm{O}_{2}\right)$ in the presence of the compound.

\section{Protein expression and purification}

The cDNA encoding for full-length OhrB (DJ41_RS22870) was chemically synthesized with codon optimization for expression in E. coli. Vector pET-28a(+) was used for protein expression. The construct was introduced into E. coli BL21 (DE3) cells for expression of recombinant proteins. The recombinant strains were cultured in LB medium $\left(10 \mathrm{gl}^{-1}\right.$ $\mathrm{NaCl}, 10 \mathrm{gl}^{-1}$ tryptone, and $5 \mathrm{gl}^{-1}$ yeast extract) at $37^{\circ} \mathrm{C}$, $220 \mathrm{rpm}$ until the OD600 reached 0.6. The cell cultures were then supplemented with $0.5 \mathrm{mM}$ isopropyl $\beta$-D-1-thiogalactopyranoside. The induced cells were further grown at $16{ }^{\circ} \mathrm{C}$ with shaking at $220 \mathrm{rpm}$ overnight to induce the expression of the recombinant protein.

To purify the recombinant proteins, the cells were harvested by centrifugation at $2000 \times g$ for $30 \mathrm{~min}$ at $4{ }^{\circ} \mathrm{C}$. The pellet was resuspended in the lysis buffer containing $50 \mathrm{mM}$ $\mathrm{NaHPO}_{4}$ (pH 8.0), $0.3 \mathrm{M} \mathrm{NaCl}, 1 \mathrm{mM}$ PMSF, $75 \mathrm{mM}$ $\mathrm{MgCl}_{2}$, and DNAase I $\left(20 \mu \mathrm{g} \mathrm{ml}^{-1}\right)$ lysed by ultrasonication. The lysate was then centrifuged at $23,000 \times g$ for $30 \mathrm{~min}$ at $4{ }^{\circ} \mathrm{C}$, and the supernatant was loaded onto a Ni-NTA column 
(a) 6D1

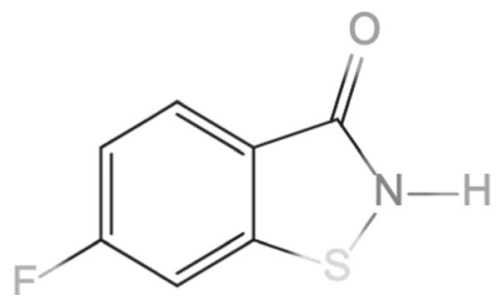

(b) BIT

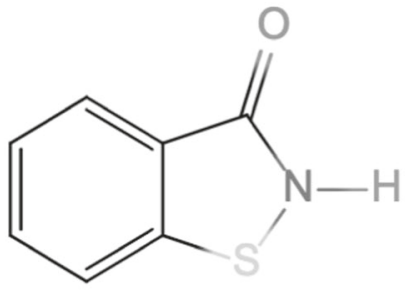

(c) Ticlatone

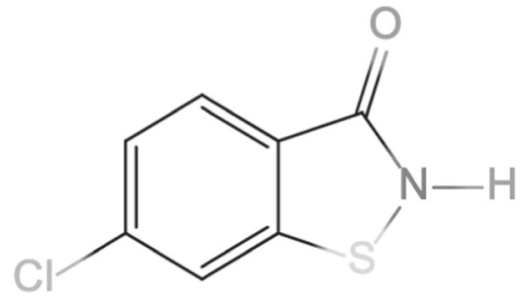

Fig. 1 Chemical structure of 6D1 and its analogs. a 6D1. b 1,2-benzisothiazolin-3-one (BIT). c Ticlatone

(Qiagen, Germany). The target protein was eluted in buffer containing $0.3 \mathrm{M} \mathrm{NaCl}, 50 \mathrm{mM} \mathrm{NaHPO} 4$ (pH 8.0), and $250 \mathrm{mM}$ imidazole. After sample analysis with SDS-PAGE, the fractions containing pure protein were pooled and concentrated to $0.5 \mathrm{ml}$, and then loaded onto a Superdex 200 increase column (GE Healthcare, USA). The protein concentration was determined using the Bradford method.

\section{Surface plasmon resonance (SPR) experiment}

OhrB were covalently immobilized to a sensor chip CM5 (291049-88, Sweden) by means of amino coupling. The running buffer used in the experiment contains $20 \mathrm{mM}$ HEPES pH 7.5, $150 \mathrm{mM} \mathrm{NaCl}, 0.05 \%$ Tween 20, 0.1\% DMSO, and the 6D1 compound was also dissolved in the running buffer. The sensor chip was washed with running buffer between each concentration. Reference runs were performed with blank (sensor chip only) and active (sensor chip with OhrB only) channel on the same sensor chip. The assay curves were constructed using serial concentrations of 6D1 of 7.5, 15, 30, 60, and $120 \mu \mathrm{M}$. The kinetic parameters of the interaction and the affinity constants were calculated using Biacore T200 evaluation software.

\section{Checkerboard titration assay}

Drug interactions between 6D1 and the bactericidal drugs were performed using a chequerboard titration assay [12]. The fractional inhibitory concentration (FIC) was calculated using the following formula: (MIC of drug $\mathrm{A}$ or $\mathrm{B}$ in combination)/(MIC of drug A or B alone). The FIC index (FICI) was determined by adding the two FICs. Synergy, antagonism, and no interaction were defined as $\mathrm{FICI} \leq 0.5$, FICI > 4.0, and FICI $=0.5-4.0$, respectively [12].

\section{Results}

\section{In vitro activity of 6D1}

We identified an active hit named 6D1 $\left(\mathrm{MIC}=1 \mathrm{mg} \mathrm{l}^{-1}\right)$ with the structure of 6-fluorobenzo[d]isothiazol-3(2H)-one,
Table 1 MICs of 6D1 that inhibited $90 \%$ of the growth of different bacterial strains

\begin{tabular}{lc}
\hline Strain & MIC $\left(\mathrm{mg}^{-1}\right)$ \\
\hline Acinetobacter baumannii ATCC19606 & 1 \\
$3 \mathrm{M}^{\mathrm{a}}$ & 10 \\
$5 \mathrm{M}^{\mathrm{a}}$ & 10 \\
Klebsiella pneumoniae ATCC13883 $^{-1}$ & 10 \\
Pseudomonas aeruginosa ATCC27853 $^{\mathrm{a}}$ ARAB-40178 & 80 \\
CRAB-42264 $^{\mathrm{b}}$ & 5 \\
CRAB-39713 $^{\mathrm{b}}$ & 5 \\
CRAB-41362 $^{\mathrm{b}}$ & 10 \\
CRAB-39351 $^{\mathrm{b}}$ & 10 \\
\hline
\end{tabular}

$C R A B$ carbapenem-resistant A. baumannii


against 6D1

${ }^{\mathrm{b}}$ Antimicrobial resistance genes are shown in Supplementary Table S1

which is similar to 1,2-benzisothiazolin-3-one (BIT) and an antifungal drug ticlatone (Fig. 1). A moderate antibacterial activity of 6D1 was also observed against in $S$. aureus $\left(\mathrm{MIC}=2.5 \mathrm{mg}^{-1}\right)$, but not against $K$. pneumoniae, and $P$. aeruginosa (MIC $\geq 5 \mathrm{mg} \mathrm{l}^{-1}$ ). Unexpectedly, MICs of 6D1 were high in CRAB clinical isolates $\left(\mathrm{MIC}=5-10 \mathrm{mg} \mathrm{l}^{-1}\right.$ ) (Table 1).

\section{OhrB mutations confer resistance to $6 \mathrm{D} 1$}

To identify the target of 6D1, we obtained two 6D1 spontaneously resistant strains (3M and 5M) from independent cultures with bacterial growth in LB broth containing $10 \times \mathrm{MIC}\left(10 \mathrm{mg} \mathrm{l}^{-1}\right)$ of $6 \mathrm{D} 1$. An increase in $\mathrm{MIC}$ indicated the resistance phenotype of $3 \mathrm{M}$ and $5 \mathrm{M}$ to 6D1 (Table 1). Compared with the wild-type (WT) parent strain A. baumannii ATCC19606, mutations located in plasmid (pMAC)-derived $o h r B$ were identified both in $3 \mathrm{M}$ and $5 \mathrm{M}$ strains, resulting in the conversion of arginine at the position 15 (Arg15) to His or Cys of OhrB (Table 2). Molecular dynamics simulations and in silico mutagenesis 
Table 2 Whole-genome sequencing identified polymorphisms within spontaneously resistant mutants of A. baumannii ATCC19606 against 6D1

\begin{tabular}{llllllll}
\hline Position of A. baumannii 19606 & Reference & 3M & 5M & Locus (DJ41_) & Gene & Product & Effect of mutation \\
\hline NZ_KL810966.1: 1913491 & G & T & - & RS13465 & - & LysR family transcriptional regulator & Asn272Lys \\
NZ_KL810966.1: 2417811 & G & - & A & RS0104185 & - & hypothetical protein & Gly951Asp \\
NZ_KL810967.1: 13770 & C & - & T & RS22870 & ohrB & organic hydroperoxide resistance protein & Arg15His \\
NZ_KL810967.1: 13771 & G & A & - & RS22870 & ohrB & organic hydroperoxide resistance protein Arg15Cys \\
\hline
\end{tabular}

Table 3 Effect of 6D1 on the susceptibility to peroxides

\begin{tabular}{lllllll}
\hline Peroxide $(\mathrm{mM})$ & Wild type & Wild type $^{\mathrm{a}}$ & $3 \mathrm{M}$ & $3 \mathrm{M}^{\mathrm{a}}$ & $5 \mathrm{M}$ & $5 \mathrm{M}^{\mathrm{a}}$ \\
\hline$t$-BHP & 0.2 & $\leq 0.005$ & 0.1 & 0.1 & 0.1 & 0.1 \\
$\mathrm{CHP}$ & 0.2 & $\leq 0.01$ & 0.2 & 0.2 & 0.2 & 0.08 \\
$\mathrm{H}_{2} \mathrm{O}_{2}$ & 0.8 & $\leq 0.1$ & 0.5 & 0.4 & 0.6 & 0.3 \\
\hline
\end{tabular}

$t$ - $\mathrm{BHP}$ tert-butyl hydroperoxide, $\mathrm{CHP}$ cumene hydroperoxide, $\mathrm{H}_{2} \mathrm{O}_{2}$ hydrogen peroxide

${ }^{a}$ The MICs of peroxides in the presence of $2.5 \mathrm{mg}^{-1}$ of $6 \mathrm{D} 1$

indicated that the corresponding Arg19 in Ohr from Xylella fastidiosa contributed to the stabilization of XfOhr in the closed state, suggesting that the mutations in $3 \mathrm{M}$ and $5 \mathrm{M}$ probably affect the function of $\mathrm{OhrB}$ [13].

\section{D1 reduces the tolerance of bacteria to peroxides}

Ohr was first described in Xanthomonas campestris. It has since been found in a number of bacterial species $[14,15]$. Owing to the Cys-based, thiol-dependent peroxidase activity, Ohr plays a central role in bacterial responses against fatty acid hydroperoxides and peroxynitrite, thus resulting in an "organic hydroperoxide resistance" phenotype [16]. Table 3 shows that the MICs of $t$-BHP, CHP, and $\mathrm{H}_{2} \mathrm{O}_{2}$ against A. baumannii ATCC19606 were at least eightfold lower when in the presence of $2.5 \mathrm{mgl}^{-1}$ of $6 \mathrm{D} 1$. The magnitude of MIC reduction coincided with the substrate preference of $\mathrm{Ohr}\left(\mathrm{H}_{2} \mathrm{O}_{2}\right.$ « $\mathrm{CHP}<t$-BHP $)$ as previously reported [16]. In contrast, against $3 \mathrm{M}$ strain, the MICs of $t$-BHP, CHP, and $\mathrm{H}_{2} \mathrm{O}_{2}$ were almost not affected by the presence of $6 \mathrm{D} 1$; whereas against $5 \mathrm{M}$ strain, the MICs of $t$-BHP, CHP, and $\mathrm{H}_{2} \mathrm{O}_{2}$ were reduced, indicating that $6 \mathrm{D} 1$ could still affect the function of $\mathrm{OhrB}$ in $5 \mathrm{M}$ strain. However, the MICs of $t$-BHP and $\mathrm{H}_{2} \mathrm{O}_{2}$ against $3 \mathrm{M}$ or $5 \mathrm{M}$ strains were slightly lower than the WT, suggesting that the $o h r B$ mutation resulted in reduced tolerance to peroxides. Moreover, growth retardation was also observed in $3 \mathrm{M}$ and $5 \mathrm{M}$ strains, but the growth of $3 \mathrm{M}$ strain was not affected by 6D1 (Fig. 2).

\section{MICs of bactericidal drugs in $3 M$ and $5 M$ strains}

With aim to identify the effects of OhrB protein function on the activity of bactericidal agents, we tested the MICs of a

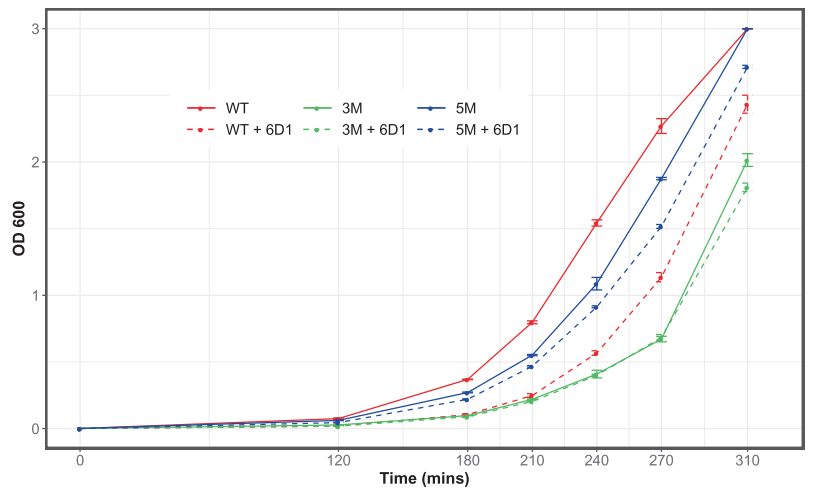

Fig. 2 Growth curve of wild-type Acinetobacter baumannii ATCC19606, $3 \mathrm{M}$, and $5 \mathrm{M}$ in the absence or presence of $0.25 \mathrm{mg} \mathrm{l}^{-1} 6 \mathrm{D} 1$

handful of bactericidal drugs against the $3 \mathrm{M}, 5 \mathrm{M}$, and WT strains. Compared with the WT, the MICs of kanamycin and gentamicin against $3 \mathrm{M}$ and $5 \mathrm{M}$ strains decreased by at least twofold, thereby suggesting the association between OhrB function and aminoglycosides activity (Table 4).

\section{Drug combinations study}

The observed changes of MIC for kanamycin and gentamicin in $3 \mathrm{M}$ and $5 \mathrm{M}$ suggest that $6 \mathrm{D} 1$ probably enhances antibacterial activity. Therefore, we used a checkerboard titration assay to identify the drug interaction of $6 \mathrm{D} 1$ with kanamycin and gentamicin in different species. Table 5 revealed a synergistic effect between 6D1 and aminoglycosides against $K$. pneumoniae and A. baumannii (FICI = 0.5). Clinical isolates of carbapenem-resistant $K$. pneumoniae (CRKP) were also selected to test the activity of 6D1 and aminoglycosides combination. The results showed that 6D1 did not reverse the antibacterial activity of kanamycin and gentamicin in CRKP containing the aminoglycosides resistance gene. However, we found that $6 \mathrm{D} 1$ enhanced the activity of kanamycin and gentamicin against a strain of CRKP that harbored the NDM-1 gene (Tables 5 and S1).

\section{The interaction between 6D1 and OhrB}

To characterize the binding of 6D1 and OhrB, we first obtained the purified His-tag-fused recombinant $\mathrm{OhrB}$ protein. The interaction between $6 \mathrm{D} 1$ and $\mathrm{OhrB}$ was confirmed by SPR analysis. It demonstrated the binding of $6 \mathrm{D} 1$ to $\mathrm{OhrB}$, with an 
association rate constant of $k_{a} 2.33 \times 10^{3} \mathrm{M}^{-1} \mathrm{~s}^{-1}$, a dissociation rate constant $k_{d} 2.28 \times 10^{-3} \mathrm{~s}^{-1}$, and an equilibrium dissociation constant $K_{D} 9.79 \times 10^{-6} \mathrm{M}$ (Fig. 3).

\section{Discussion}

Bacteria have evolved complex mechanisms to detoxify reactive oxygen species and thus strictly control $\mathrm{H}_{2} \mathrm{O}_{2}$ levels. A 9540-bp plasmid pMAC carried by A. baumannii ATCC19606 that contains an OhrB coding region conferred bacterial resistance to organic peroxide-generating compounds CHP and $t$-BHP was reported in 2006 [17]. In this study, a whole-cell assay revealed a compound 6D1, which imparted inhibitory effects on A. baumannii ATCC19606 and had a similar structure to BIT and the antifungal ticlatone. The mutation site of the spontaneously resistant mutant and SPR result suggested that the interaction between 6D1 and pMAC-derived OhrB. A previous study suggested that cellular thiol groups are major targets of BIT [18]. The antimicrobial activity of 6D1 on $K$. pneumoniae was also reported previously [19]. Therefore, it provides a rationale that $6 \mathrm{D} 1$ acts on the thiol groups, thereby exerting

Table 4 MICs of different classes of antibiotics that inhibited $90 \%$ of bacterial growth

\begin{tabular}{llll}
\hline Antibiotic & Wild type $\left(\mathrm{mg}^{-1}\right)$ & $3 \mathrm{M}\left(\mathrm{mg}^{-1}\right)$ & $5 \mathrm{M}\left(\mathrm{mg}^{-1}\right)$ \\
\hline Ceftazidime & 8 & 8 & 8 \\
Meropenem & 4 & 4 & 4 \\
Kanamycin & 16 & 4 & 8 \\
Gentamicin & 16 & 8 & 8 \\
Ofloxacin & 0.25 & 0.25 & 0.25 \\
Polymyxin B & 2 & 2 & 2 \\
Tetracycline & 0.3125 & 0.625 & 0.625 \\
Rifampicin & 1.56 & 1.56 & 1.56 \\
Chloramphenicol & 64 & 64 & 64 \\
\hline
\end{tabular}

an antibacterial effect. However, our study found that 6D1 can interact with Ohr. This is concordant with our result that 6D1 sensitizes A. baumannii ATCC19606 to CHP and $t$-BHP. Although $\mathrm{Ohr}$ is not an essential gene, and therefore cannot be the primary target of an antibiotic, the inhibitory effect of Ohr still suggests the potential application of 6D1, given its important role in bacterial resistance to peroxides.

In addition to developing antibiotics with new chemical structures and acting mechanisms, antibiotic adjuvants offer an alternative approach to combat resistance [20]. In this study, 6D1 was found to impart an inhibitory effect on OhrB, and thus it is reasonable to use this as an adjuvant in combination with other antibiotics that induce bacteria to produce hydroperoxides. In addition, a previous study showed that all bactericidal antibiotics induce protective responses to reactive oxygen species [21]. This suggests the potential of 6D1 as an adjuvant for bactericidal drugs. However, our data showed that 6D1 only enhances the activity of the aminoglycosides,

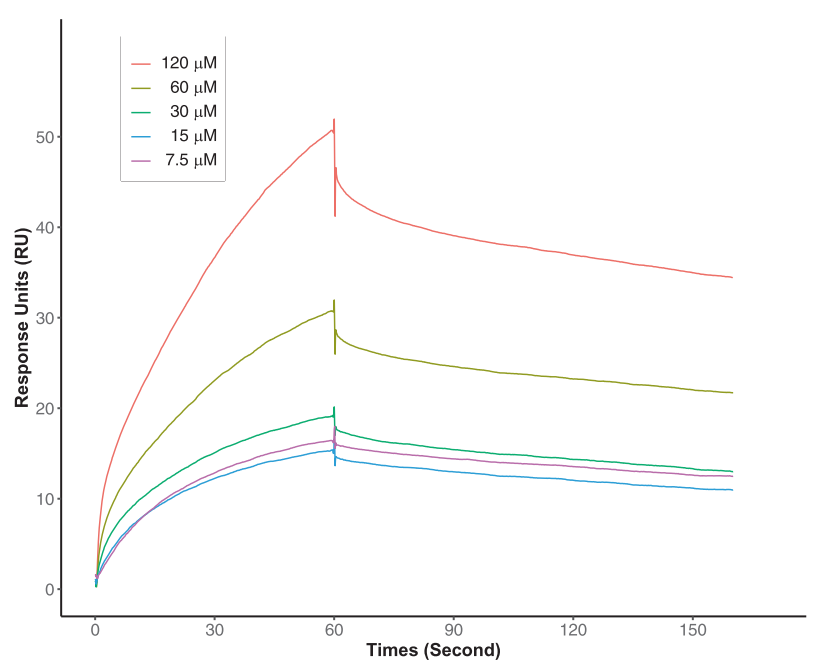

Fig. 3 Surface plasmon resonance analysis of the interaction between $6 \mathrm{D} 1$ and $\mathrm{OhrB}$. The $K_{D}$ values were determined from the ratio between the kinetic rate constants $\left(k_{a} k_{d}^{-1}\right)$
Table 5 Potency of 6D1 in combination with kanamycin and gentamicin against different species

\begin{tabular}{|c|c|c|c|c|c|c|c|}
\hline \multirow[t]{2}{*}{ Strain } & \multirow{2}{*}{$\begin{array}{l}\text { MIC of 6D1 } \\
\left(\mathrm{mg}^{-1}\right)\end{array}$} & \multicolumn{3}{|c|}{ Kanamycin } & \multicolumn{3}{|c|}{ Gentamicin } \\
\hline & & $\begin{array}{l}\mathrm{MIC} \\
\left(\mathrm{mg} \mathrm{l}^{-1}\right)\end{array}$ & $\begin{array}{l}\text { Fold change } \\
\left(\mathrm{mg} \mathrm{l}^{-1}\right)^{\mathrm{a}}\end{array}$ & FICI & $\begin{array}{l}\mathrm{MIC} \\
\left(\mathrm{mg} \mathrm{l}^{-1}\right)\end{array}$ & $\begin{array}{l}\text { Fold change } \\
\left(\mathrm{mg} \mathrm{l}^{-1}\right)^{\mathrm{b}}\end{array}$ & $\overline{\text { FICI }}$ \\
\hline A. baumannii 19606 & 1 & 16 & $4(0.25)$ & 0.5 & 16 & $4(0.25)$ & 0.5 \\
\hline $3 \mathrm{M}$ & 10 & 4 & $2(0.625)$ & 0.5625 & 8 & $2(2.5)$ & 0.75 \\
\hline $5 \mathrm{M}$ & 10 & 8 & $4(2.5)$ & 0.5 & 8 & $2(2.5)$ & 0.75 \\
\hline К. pneumoniae 13883 & 10 & 8 & $4(2.5)$ & 0.5 & 8 & $4(2.5)$ & 0.5 \\
\hline CRKP-34978 & 20 & 16 & $4(5)$ & 0.5 & 8 & $4(5)$ & 0.5 \\
\hline CRKP-36472 & 10 & $>256$ & - & - & $>256$ & - & - \\
\hline CRKP-38218 & 10 & $>256$ & - & - & $>256$ & - & - \\
\hline CRKP-42644 & 20 & $>256$ & - & - & $>256$ & - & - \\
\hline CRKP-40847 & 10 & $>256$ & - & - & $>256$ & - & - \\
\hline
\end{tabular}

${ }^{\mathrm{a}}$ The concentration of $6 \mathrm{D} 1$ used in the combination

${ }^{\mathrm{b}}$ Antimicrobial resistance genes are shown in Supplementary Table S1 
kanamycin, and gentamicin, but not non-aminoglycoside. This may be related to the reactivity order of Ohr to different peroxides, in which it mainly modulates the levels of fatty acid hydroperoxides and peroxynitrite [16]. Because the synergistic effect of 6D1 is achieved by inhibiting OhrB, it is not surprising that $6 \mathrm{D} 1$ was not effective on drug-resistant strains that harbored aminoglycosides resistance genes, such as the 16s rRNA methylase enzyme $r m t B$. Notably, the combination of 6D1 with kanamycin and gentamicin were effective on the CRKP strain carrying NDM-1. A recent study has shown that in Northeast China, the aminoglycoside resistance gene $r m t B$ was detected in $96.61 \%$ of KPC-2producing CRKP and in $21.74 \%$ of NDM-1-producing CRKP, indicating the potential combinative application of aminoglycosides and a peroxidase inhibitor, such as 6D1 in about $80 \%$ of NDM-1-producing CRKP [22].

The clinical use of aminoglycosides has been limited by its well-known toxicity and side effects, such as ototoxicity. Our study revealed the feasibility of enhancing the activity of aminoglycosides by inhibiting the detoxification ability of bacteria to peroxides, thereby providing a new target and strategy for the development of aminoglycosides enhancers in the near future.

Funding The International Collaborative Research Fund (GJHZ201 80413181716797) and Free Inquiry Fund (JCYJ20180305163929948) of Shenzhen Science and Technology Innovation Commission supported this study.

\section{Compliance with ethical standards}

Conflict of interest The authors declare that they have no conflict of interest.

Publisher's note Springer Nature remains neutral with regard to jurisdictional claims in published maps and institutional affiliations.

\section{References}

1. US Department of Health and Human Services: Centers for Disease Control and Prevention. Antibiotic Resistant Threats In The United States. Centers for Disease Control and Prevention; 2019. https://www.cdc.gov/drugresistance/biggest-threats.html.

2. Magill SS, et al. Multistate point-prevalence survey of health careassociated infections. N. Engl J Med. 2014;370:1198-208.

3. Garnacho-Montero J, et al. Task force on management and prevention of Acinetobacter baumannii infections in the ICU. Intensive Care Med. 2015;41:2057-75.

4. Giamarellou H, Poulakou G. Pharmacokinetic and pharmacodynamic evaluation of tigecycline. Expert Opin Drug Metab Toxicol. 2011;7:1459-70.
5. Xie R, Zhang XD, Zhao Q, Peng B, Zheng J. Analysis of global prevalence of antibiotic resistance in Acinetobacter baumannii infections disclosed a faster increase in OECD countries. Emerg Microbes Infect. 2018;7:31.

6. Qureshi ZA, et al. Colistin-resistant Acinetobacter baumannii: beyond carbapenem resistance. Clin Infect Dis. 2015;60: 1295-303.

7. Hartzell JD, et al. Nephrotoxicity associated with intravenous colistin (colistimethate sodium) treatment at a tertiary care medical center. Clin Infect Dis. 2009;48:1724-8.

8. Connors KP, et al. Phase I, open-label, safety and pharmacokinetic study to assess bronchopulmonary disposition of intravenous eravacycline in healthy men and women. Antimicrob Agents Chemother. 2014;58:2113-8.

9. Poulakou G, Lagou S, Karageorgopoulos DE, Dimopoulos G. New treatments of multidrug-resistant Gram-negative ventilatorassociated pneumonia. Ann Transl Med. 2018;6:423.

10. World Health Organization. Antibacterial agents in clinical development: an analysis of the antibacterial clinical development pipeline, including tuberculosis. World Health Organization; 2017. https://apps.who.int/iris/handle/10665/258965.

11. Bankevich A, et al. SPAdes: a new genome assembly algorithm and its applications to single-cell sequencing. J Comput Biol. 2012;19:455-77.

12. Odds FC. Synergy, antagonism, and what the chequerboard puts between them. J Antimicrob Chemother. 2003;52:1.

13. Piccirillo E, et al. Structural insights on the efficient catalysis of hydroperoxide reduction by Ohr: Crystallographic and molecular dynamics approaches. PLoS One. 2018;13:e0196918.

14. Mongkolsuk S, Praituan W, Loprasert S, Fuangthong M, Chamnongpol S. Identification and characterization of a new organic hydroperoxide resistance (ohr) gene with a novel pattern of oxidative stress regulation from Xanthomonas campestris $p v$. phaseoli. J Bacteriol. 1998;180:2636-43.

15. Atichartpongkul $\mathrm{S}$, et al. Bacterial $\mathrm{Ohr}$ and OsmC paralogues define two protein families with distinct functions and patterns of expression. Microbiology. 2001;147:1775-82.

16. Alegria TG, et al. Ohr plays a central role in bacterial responses against fatty acid hydroperoxides and peroxynitrite. Proc Natl Acad Sci USA. 2017;114:E132-41.

17. Dorsey CW, Tomaras AP, Actis LA. Sequence and organization of pMAC, an Acinetobacter baumannii plasmid harboring genes involved in organic peroxide resistance. Plasmid. 2006;56:112-23.

18. Cóllier PJ, et al. Chemical reactivity of some isothiazolone biocides. J Appl Bacteriol. 1990;69:578-84.

19. Carmellino ML, Pagani G, Pregnolato M, Terreni M, Pastoni F. Antimicrobial activity of fluorinated 1,2-benzisothiazol-3(2H)ones and 2,2'-dithiobis(benzamides). Eur J Med Chem. 1994;29: 743-51.

20. Wright GD. Antibiotic adjuvants: rescuing antibiotics from resistance. Trends Microbiol. 2016;24:862-71.

21. Kohanski MA, Dwyer DJ, Hayete B, Lawrence CA, Collins JJ. A common mechanism of cellular death induced by bactericidal antibiotics. Cell. 2007;130:797-810.

22. Lin L, Xiao X, Wang X, Xia M, Liu S. In vitro antimicrobial susceptibility differences between carbapenem-resistant KPC-2producing and NDM-1-producing Klebsiella pneumoniae in a Teaching hospital in Northeast China. Micro Drug Resist. 2020;26:94-99. 